matters. The Supreme Court may uphold, vary, set aside or order the retrial of the case as it deems fit.

According to the law, on discharge, the patient should be provided with community and home services as required. There is, however, no provision for a compulsory community care order.

\section{Discussion}

The law applies to individuals of all ages, and there is no separate provision for minors in the compulsory admission rules. Parents have substantial input in the decision-making for compulsory admission of minors, although the latter is very uncommon. Children and adolescents are admitted to a specific in-patient facility located at a general hospital (the Makarion Hospital) and not the secure psychiatric centre, which does not cater for minors.

With respect to criteria for compulsory admission, it is not made explicit whether such an admission can be considered in the absence of any risk to the safety of the self or others. However, the criterion 'for the protection of self' could be interpreted as meaning the protection of health, preventing deterioration and ensuring well-being. It is not known how often the Act may be used in this way; anecdotally, it appears that doctors prefer not to do so.
Every state hospital is required to appoint a Patient's Rights Officer, and there are designated 'mail boxes' easily accessible to patients and relatives in which to place any complaints, which are collected by a member of the MHC. Leaflets explaining the rights of patients detained under the MHA are made available to them and are also displayed on a wall in all wards, but there is no independent advocacy service other than the above arrangements.

It is not possible to know how the law is applied in practice, as there are no studies as yet specifically examining such issues. Studies are also needed to examine the effectiveness of the supervision by the MHC.

\section{References}

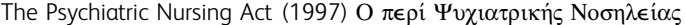
Nómos (N. 77(I)/1997 (Law on Psychiatric Care). House of Representatives; Cyprus.

WHO (2008) Suicide rates by gender and age, Cyprus. http://www. who.int/mental_health/media/cyprus.pdf

WHO (2015) World health rankings 2015. www.worldlifeexpectancy. com/country-health-profile/cyprus

World Bank (2017) World Bank list of economies. databank. worldbank.org/data/download/site-content/CLASS.xls
PSYCHIATRY UNDER RESTRICTIVE CONDITIONS

\title{
Restrictive environments: the challenge of implementing of Brunei's 2014 Mental Health Order
}

\author{
Hilda Ho
}

Ministry of Health, Bandar Seri Begawan, Brunei Darussalam: email hilda.ho@moh.gov.bn

Acknowledgements: I thank Dr Rifai Farid for reviewing the initia drafts of the paper.

Conflicts of interest. The author is Head of Psychiatry at Raja Isteri Pengiran Anak Saleha (RIPAS) Hospital, Ministry of Health, Brunei Darussalam. The views expressed in this paper are her own and do not necessarily represent those held by the Ministry of Health.

doi:10.1192/bji.2017.19
In 2014, Brunei implemented the Mental Health Order, which replaced the 1929 Lunacy Act. This new legislation was designed to improve the treatment of mental disorders and to protect the rights and welfare of people with mental disorders. This short report describes the experience and challenge of implementing the new legislation, with a particular focus on the use of involuntary treatment and the restrictions imposed on people with mental disorders despite the new legislative framework.

Brunei Darussalam is a small country (population 406000 ) in Southeast Asia, which scores highly on economic, health and social indicators (United Nations, 2015). It has a dual legal system consisting of Islamic Syariah law and civil law. Many of its civil laws predate independence and are based on English common law. Healthcare services are predominantly provided by the government free of charge to all citizens and permanent residents.

\section{The aims of the new legislation}

The development, aims and content of the 2014 Mental Health Order (available at http://www. agc.gov.bn) have been described previously (Ho, 2016). One of its main aims was to improve the process of involuntary treatment and to safeguard the patient's rights throughout the process. The guiding principles included the preservation of individual autonomy and the use of 'least restrictive' treatment options, while acting in the best interests of patients. Statutory reviews of involuntary treatment were built in, and an appeals process was introduced. It was hoped that the 
(c) The Author 2018. This is an Open Access article, distributed under the terms of the Creative Commons AttributionNonCommercial-NoDerivatives licence (http://creativecommons. org/licenses/by-nc-nd/4.0/), which permits non-commercial re-use, distribution, and reproduction in the original work is unaltered and is properly cited. The written permission of Cambridge University Press must be obtained for commercial re-use or in order to create a derivative work. legislation would provide a framework to protect the rights and welfare of people with mental disorders. It was expected that proper implementation would require a systemic change in attitude and practice by multiple agencies, including healthcare providers, criminal justice services, social welfare services and the wider community. Preparation for this included consultation, training and dissemination of information. Standardised forms and multi-agency protocols were prepared to ensure consistency; a code of practice was published (Ministry of Health, 2014); and a board of visitors was appointed for each in-patient psychiatric facility to inspect facilities and to review patients given long-term involuntary treatment.

\section{Implementation}

Implementation of the new legislation commenced on 1 November 2014 in all hospitals and health services. Quantitative data comparing involuntary and voluntary admissions for the first-year cohort of patients have been evaluated and reported elsewhere (Ho, 2017a). Involuntary admissions were found to have substantially exceeded voluntary admissions.

\section{Psychiatric systems}

In recent years, mental health services in Brunei have undergone a period of development (Ho, 2014). However, Brunei does not have any designated secure forensic or high-dependency psychiatric facilities. In-patient psychiatric treatment is provided in two general psychiatric wards. Individual locked seclusion rooms are the highest level of physical restriction, followed by involuntary in-patient treatment in two locked general psychiatric wards. Until recently, the psychiatric facility in the capital city, which served three-quarters of the country's population, was in a dilapidated state, with cramped accommodation in a mixed psychiatric ward within a general hospital. Many patients refused admission owing to the poor state of this facility. Unfortunately, some patients had to be admitted involuntarily owing to the lack of an alternative in-patient facility. After several years of effort and planning, a specially refurbished community psychiatry centre was opened in May 2017. In-patient, outpatient and rehabilitation services now operate from a pleasant, spacious site with low-rise buildings and gardens. Supported accommodation is located nearby. There is a smaller psychiatric ward in a district general hospital located 110 $\mathrm{km}$ from the capital city, which was also refurbished recently in 2011. It is hoped that the provision of better facilities will improve the quality and acceptability of psychiatric treatment. Formal evaluation will be required to measure patient satisfaction and quality of care.

Community treatment orders are also available for involuntary treatment after discharge from in-patient care. However, these have rarely been used since implementation (Ho, 2017a). Owing to the lack of secure forensic psychiatric facilities, offenders with mental disorders are remanded in prison and receive treatment through a visiting psychiatric clinic. There has been some expectation from criminal justice agencies that offenders with mental disorders would be dealt with by general psychiatric facilities, despite the lack of secure facilities. The situational challenges of managing offenders with mental disorders in Brunei have been described elsewhere (Ho, 2017b).

\section{Challenges to the use of appropriate and 'least restrictive' treatment}

\section{Culture}

Bruneian society is by and large respectful of authority and non-confrontational in terms of private and public interaction. The culture is highly family oriented and structured according to gender and age. People are expected to conform to societal norms in terms of behaviour and lifestyle. Any kind of unexpected behaviour may be suspected to be a result of black magic or spiritual assault. Sufferers are usually taken to a spiritual healer for 'treatment', whether or not they consent. Mental disorders are often considered only when the affected person becomes aggressive. This is because physical aggression is often regarded as a mental health problem, and there is an expectation of intervention by mental health services. Alternative explanations for aggressive behaviour, such as intoxication, goal-directed behaviour or personality, can be less acceptable. The previous Lunacy Act did not contain any definition of mental disorder, or any provision for voluntary treatment. Its purpose was to remove people with mental disorders from society, to be placed in care by the court. With the introduction of the new legislation, voluntary treatment is expected where capacity is intact. Unfortunately, there is often an assumption of incapacity to make decisions regarding mental health treatment. In some cases, there may be family pressure to 'lock up' disruptive family members whether or not there is a mental disorder.

\section{Multi-agency services}

There are few alternatives to in-patient psychiatric treatment. Mental healthcare provision in primary healthcare settings is restricted by limited expertise and a lack of psychotropic medications. General community mental health services are not resourced to respond to acute crises. There are no intensive home-based treatment programmes available to support acutely ill patients at home or facilitate early discharge. A small population and lack of human resources are significant limiting factors in the establishment of specialist community services in Brunei. Often, involuntary treatment in hospital is the only safe treatment option available for acutely ill patients and a smaller number of patients with chronic disorders. 
Unfortunately, there is limited multi-agency interest in the setting up of care packages, such as housing, criminal justice supervision and social welfare assistance, for those with complex needs. There are no non-governmental organisations that provide coordinated community care. These deficiencies have contributed to a reliance on involuntary hospital treatment to 'contain' problems rather than to ensure optimal quality of life in the community. In recent years, a few voluntary organisations have started to contribute periodically to mental health services in the form of organising recreational activities, providing household materials and helping with home repairs. It will take some time for these groups to expand their scope of work and expertise. The expansion of coordinated care outside the government sector is crucial to improve the community integration of patients and reduce stigma.

\section{Independent advocacy and legal support}

Brunei has no services providing independent advocacy or legal support for people with mental disorders. This may reflect the stigma of having a mental disorder and the secrecy in dealing with it among individuals and families. There is no legal aid funding available for patients who wish to appeal their involuntary treatment. Patients are not routinely informed of the procedures for appeal, and there is no statutory requirement for hospitals to display this information. Only one patient so far has formally appealed their involuntary treatment. Given the nonconfrontational culture and the lack of independent support, it is likely that many patients are discouraged or do not have the opportunity to appeal.

\section{The way forward}

For a long time, the treatment of mental disorders in Brunei was overly restrictive. The 2014 Mental Health Order initiated a change in policy and practice for the protection of people with mental disorders. Nevertheless, ensuring systemic change in a society requires the consistent engagement of multiple agencies and the wider community. Although Brunei has a unique culture, the general principles laid out by the World Health Organization (WHO) in its Mental Health Action Plan 2013-2020 (WHO, 2013) could be used to inform further work. The WHO QualityRights Tool Kit (WHO, 2012) may help guide the assessment and improvement of human rights standards in care facilities.

\section{Conclusions}

The implementation of the Mental Health Order has initiated change and provided a framework for the protection of the rights and welfare of people with mental disorders. However, the greater challenge remains of engaging communities and empowering patients, to ensure the appropriate and dignified treatment of people with mental disorders without placing unnecessary restrictions on their lives.

\section{References}

Ho H. (2014) Mental healthcare in Brunei Darussalam. International Psychiatry, 11(4), 100-102.

Ho H. (2016) Brunei Darussalam's new Mental Health Order. BJPsych International, 13(2), 38-40.

Ho H. (2017a) Review of the implementation of Brunei Darussalam's new Mental Health Order in a psychiatric ward in RIPAS Hospital. Brunei International Medical Journal, 13(2), 44-50.

Ho H. (2017b) The management of mentally disordered offenders within the criminal justice system in Brunei Darussalam. BJPsych International, 14(3), 72-74.

Ministry of Health (2014) Code of Practice, Mental Health Order, 2014, Brunei Darussalam. Ministry of Health.

United Nations (2015) World Statistics Pocketbook Country Profile: Brunei Darussalam. UN. Available at http://data.un.org/CountryProfile. aspx?crName=Brunei\%20Darussalam (accessed July 2017).

World Health Organization (2012) WHO Quality Rights Toolkit. WHO. Available at http://apps.who.int/iris/bitstream/10665/70927/3/ 9789241548410_eng.pdf (accessed July 2017).

World Health Organization (2013) Mental Health Action Plan 2013 2020. Resolution WHA 66/8. WHO. Available at http://www.who.int/ mental_health/publications/action_plan/en/ (accessed July 2017).

\title{
SPECIAL PAPER \\ Mental capacity in Colombia: a comparison with the UK
}

\author{
Juan P. Borda, ${ }^{1}$ Ricardo Tamayo ${ }^{2}$ and Gareth Owen ${ }^{3}$
}

${ }^{1}$ Corporación Universitaria Empresarial Alexander von Humboldt, Faculty of Medicine, Armenia, Colombia. Email: juanpablobordab@gmail.com

${ }^{2}$ Consultant Psychiatrist, Instituto Nacional de Medicina Legal y Ciencias Forenses, Bogotá, Colombia
Several international proclamations in the last decades have advocated for the dignity and autonomy of persons with mental disorders. Few discussions have been generated regarding the implication of this transition in low- and middle-income countries. The objective of this publication is to review how the concept of mental capacity has been defined in Colombian law. We then briefly compare the Colombian and UK situations and propose a few points of discussion, addressing some difficulties and challenges of 